\title{
Anti-inflammatory effects of hyaluronan in arthritis therapy: Not just for viscosity
}

REVIEW

This article was published in the following Dove Press journal:

International Journal of General Medicine

28 April 2009

Number of times this article has been viewed

\section{Kayo Masuko' \\ Minako Murata² \\ Kazuo Yudoh ${ }^{2}$ \\ Tomohiro Kato' \\ Hiroshi Nakamura ${ }^{3}$ \\ 'Department of Biochemistry; \\ ${ }^{2}$ Institute of Medical Science, \\ St. Marianna University School of Medicine, Kawasaki-shi, Kanagawa, Japan; ${ }^{3}$ Department of Joint Disease and Rheumatism, Nippon Medical School, Bunkyo-ku, Tokyo, Japan}

Correspondence: Kayo Masuko Department of Biochemistry, St. Marianna University School of Medicine, 2-16-I Sugao, Miyamae-ku, Kawasaki-shi, Kanagawa 216-85II, Japan Tel +8I 4497781 II

Fax +8I 449767553

Email k_msk@mac.com
Abstract: Hyaluronic acid (HA) has been widely used for viscosupplementation of diseased or aged articular joints. However, recent investigations have revealed the active anti-inflammatory or chondroprotective effect of HA, suggesting its potential role in attenuation of joint damage. In particular, interactions between $\mathrm{HA}$ and other inflammatory mediators are attracting interest. This review summarizes several aspects of recent investigations of the anti-inflammatory effects of HA in arthritis.

Keywords: hyaluronan, inflammation, chondroprotection

\section{Introduction}

Intra-articular administration of hyaluronic acid (HA; hyaluronan) is a widely applied treatment for osteoarthritis (OA) (reviewed by Simon and Strand). ${ }^{1}$ Although HA injection provides relief from pain, albeit transiently, its mechanism of action has not been fully clarified. In a recent recommendation on the management of hip and knee OA by the Osteoarthritis Research Society International (OARSI), ${ }^{2}$ the strength of recommendation (SOR) of intra-articular HA was calculated to be relatively low (64\%), whereas that of nonsteroidal anti-inflammatory drugs (NSAIDs) for oral administration as analgesics was $92 \%-93 \%$.

The effect of HA in OA has often been attributed to its potential use in viscosupplementation, ie, supplementation of viscosity; however, the benefits of HA from "some other action and not by its biomechanical properties" is also suggested (reviewed by Neustadt and colleagues). ${ }^{3}$ More specifically, a direct effect of HA against inflammation or cartilage degradation in OA has also been suggested.

In addition to its use in OA, intra-articular injection of HA has been used in patients with inflammatory arthritis such as rheumatoid arthritis (RA). In some reports, it is indicated that HA has beneficial effects on inflammatory arthritis. ${ }^{4-6}$

This study briefly reviews the recent findings related to the function of HA, focusing on its reported effects in chondrocytes, not only in supplementing viscosity, but also as a potential anti-inflammatory anti-arthritic agent. Since it is well-known that oligosaccharide hyaluronan is inversely involved in inflammation, ${ }^{7}$ whenever HA is mentioned in this paper, it should be understood that the reference is to high molecular weight hyaluronan.

\section{Biochemistry of HA}

Hyaluronic acid is a polysaccharide composed of repeating disaccharide units, ie, 1,4-glucuronic acid (GlcUA) and 1,3-N-acetylglucosamine (GlcNAC). HA belongs

submit your manuscript | www.dovepress.co 
to the group of glycosaminoglycans, but unlike chondroitin sulfate or keratan sulfate, HA is not sulfated. HA is synthesized by bioactivity of hyaluronan synthase (HAS), which has been reported to have three isoforms (HAS1, HAS2, and HAS3) in humans. ${ }^{8}$

HA is widely distributed but mainly localized to the extracellular matrix and body fluid. It contributes to the viscoelasticity of the fluid and elasticity in connective tissues, which absorb mechanical stress, for example, between cartilage and cartilage surfaces. ${ }^{3}$

In addition to this property of providing viscoelasticity, recent investigations have continued to reveal the variety of actions of HA on cartilage. For example, in normal diarthrodial joints, HA not only works as a mechanical/physical barrier, but also has anti-inflammatory and analgesic functions. ${ }^{3}$ As for pain suppression, it has been shown that HA attenuates prostaglandin- or bradykinin-induced pain in experimental OA animals..$^{9,10}$

HA binds to the signal-transducing receptor molecules CD44 and receptor for HA-mediated motility (RHAMM; reviewed by Taylor and colleagues). ${ }^{11}$ In this regard, CD44 is the primary receptor of HA, and has also been found to bind with other matrix components, including collagen, chondroitin sulphate, and osteopontin. ${ }^{11-13}$ Binding of the components to CD44 leads to modulation of cellular functions; ${ }^{14-16}$ for example, HA is reported to inhibit proteoglycan degradation and anti-Fas-induced apoptosis of chondrocytes through CD44 expressed on the cells. ${ }^{17,18}$

\section{HA suppresses inflammatory responses in articular joints HA suppresses MMPs and ADAMTS}

In inflammatory or degenerative joints, cartilage matrix is degraded by the action of an array of proteinases. In particular, the families of matrix metalloproteinases (MMP) and a disintegrin and metalloproteinase with thrombospondin motifs (ADAMTS) are mainly responsible for cartilage degradation. More specifically, the collagenases (MMP-1 and MMP-13) and stromelysin-1 (MMP-3) play a pivotal role in collagen degradation, whereas the aggrecanases (ADAMTS) induce aggrecan degradation. These proteinases are often induced by inflammatory stimuli, such as interleukin-1 (IL-1) or tumor necrosis factor- $\alpha$ (TNF- $\alpha$ ), which are activated by inflammation or mechanical or physical stress in joints. ${ }^{19,20}$ Subsequently, in an inflamed joint, cartilage exposed to the induced ADAMTS-4 and ADAMTS-5 would release $\mathrm{HA}$, indicating deterioration of matrix integrity, ${ }^{21}$ this loss of HA from the cartilage matrix is thought to result in the progression of cartilage degradation. On the other hand, it has been demonstrated that HA can enhance synthesis of chondroitin sulfate and proteoglycans, ${ }^{22,23}$ and reduce the production and activity of MMPs and ADAMTS. ${ }^{5,24,25}$

IL-1 is considered to be the dominant catabolic cytokine present in the inflamed articular joints. In this regard, HA is reported to counteract the catabolic process induced by IL-1. For example, Fioravanti and colleagues stimulated human chondrocytes under hydrostatic pressure and showed that HA (MW 500-730 kDa) abrogates a decrease in proteoglycan and an increase in nitric oxide (NO) levels, both induced by IL-1. In a study by Karna and colleagues, HA (500-730 kDa) was found to counteract the effect of IL-1, as HA inhibited IL-1-induced downregulation of type II collagen mRNA expression. ${ }^{26}$ Julovi and colleagues ${ }^{27}$ used $800-\mathrm{kDa}$ HA to assess the inhibitory effect of HA on IL-1 $\beta$-induced MMPs in cartilage and found that the $800-\mathrm{kDa}$ HA penetrated cartilage tissue, localized in the pericellular matrix around chondrocytes, and bound with CD44 on chondrocytes, leading to the suppression of IL-1 $\beta$-stimulated MMP production by chondrocytes. In this context, we found that HA $(800 \mathrm{kDa}$ and $1900 \mathrm{kDa}$ ) suppressed IL-1-induced MMP-1 production both at protein and mRNA levels in cultured human chondrocytes, and this effect was cancelled by treatment with anti-CD44 antibody. ${ }^{28}$

As for synovial fibroblasts, Shimizu and colleagues ${ }^{29}$ reported that HA inhibited TNF- $\alpha$ - and IL-1-induced MMP-1 production from rheumatoid synovial fibroblasts. For this effect, HA was suggested to bind with CD44 expressed in synovial fibroblasts. More recently, the interaction between synovial fibroblasts and HA via intracellular adhesion molecule-1 (ICAM-1) was reported by Hiramitsu and colleagues. ${ }^{29}$ The mechanism of this inhibition was speculated to occur through HA binding to ICAM-1, leading to downregulation of nuclear factor- $\kappa \mathrm{B}(\mathrm{NF}-\kappa \mathrm{B})$ and $\mathrm{p} 38$ mitogenactivated protein kinase (MAPK) phosphorylation.

In addition to the effects shown against MMPs, a recent report by Yatabe and colleagues indicated the inhibition of ADAMTS4 (aggrecanase-1) expression in human chondrocytes by HA (2700 kDa) through the CD44 and ICAM-1 signaling pathways. ${ }^{25}$ These findings collectively suggest a therapeutic potential of HA against matrix breakdown in arthritic cartilage.

\section{Plasmin/plasminogen system and HA}

Fibrinolysis is one of the major pathological changes occurring in arthritic joints, in which the plasminogen system is known to play a key role. The balance between plasminogen 
activator (PA) and plasminogen activator inhibitor (PAI) determines the direction in which plasminogen is activated into plasmin, which in turn further promotes fibrinolysis by activating proteinases, including gelatinases and other MMPs. PA occurs in two forms: urokinase-type PA (uPA) and tissue-type PA (tPA).

It has been suggested that hyaluronan modulates the PA system in articular joints. For example, Nonaka and colleagues $^{30}$ reported that HA suppressed the secretion of u-PA and PAI-1 from OA synovial fibroblasts. Further, it was found that the receptor for u-PA (u-PAR) was expressed in the synovium in both OA and RA and that the expression was attenuated by treatment with HA. ${ }^{30}$ These results suggested that HA could suppress fibrinolytic activity mediated by the UPA and UPAR systems. More recently, Hsieh et al measured the levels of MMP-2, MMP-9, u-PA, and PAI-1 in a series of chondral, meniscal, and synovial cultures of early OA after treatment with or without three HA products with different molecular weights (MW), and demonstrated that all of the HA products could decrease the secretion of MMP-2 and MMP-9. They suggested that HA with high MW might be more effective in inhibiting MMP-2, MMP-9, $\mathrm{u}-\mathrm{PA}$, and PAI-1 expression. ${ }^{31}$ Further investigation on the crosstalk between PA/PAI and HA would provide important clues to the protection of cartilage degradation by these proteinases.

\section{Hyaluronan and cyclooxygenase}

Acidic NSAIDs are a major subset of anti-inflammatory drugs that inhibit the activity of cyclooxygenase (COX), leading to suppression of prostaglandins and leukotrienes in inflamed tissue. In this regard, interaction has been demonstrated between HA or its fragment and COX-2 expression in a variety of cells. ${ }^{32-35}$ Several studies showed upregulation of COX-2 expression by HA. For example, HA (200-kDa) was reported to increase the expression of glomerular COX-2 in rat renal glomerular cells ${ }^{34}$ and in human vascular endothelial cells, ${ }^{36}$ respectively. The effect of HA has been reported to be mimicked by an engagement of CD44 (an HA receptor). ${ }^{35,36}$ Further, Stuhlmeier reported prostaglandin $\mathrm{E}_{2}\left(\mathrm{PGE}_{2}\right)$ as a potent activator of HAS1 in IL-1-treated type-B-synoviocytes. ${ }^{37}$ In that study, the author tested a series of eicosanoids and found that $\mathrm{PGE}_{2}$, and to a lesser extent, $\mathrm{PGI}_{2}$ and thromboxane $\mathrm{A}_{2}$ analogues activated HAS1 expression in synoviocytes after IL-1 treatment, whereas the lipoxygenase product leukotriene B4 had no effect on HAS1 induction. Therefore, HA may upregulate $\mathrm{COX}-2$, causing the activated $\mathrm{COX}-2$ to induce
$\mathrm{PGE}_{2}$, which would then activate HAS1 through a positive feedback mechanism (Figure 1). Conversely, Mitsui and colleagues demonstrated an inhibitory effect of HA on the expression of COX-2 mRNA in IL-1-stimulated subacromial synovial fibroblasts. ${ }^{33}$ In that study, however, HA with a molecular weight of $90 \times 10^{4}$ was used; therefore, the different effect of HA might have been due to the difference in the molecular weight. In addition, Kinugasa and colleagues ${ }^{40}$ reported that inhibition of COX-2 using a COX inhibitor or COX-2 antisense oligonucleotide in a tumor cell line resulted in downregulation of CD44 expression. $^{38}$

Collectively, the HAS/HA system and COX/PG system may have a regulatory network via CD44, which would be regulated differently by each HA component present in each cell type, and further modulated by the presence of inflammatory cytokines and mediators (Figure 1). Still, inconsistency among studies concerning the interaction between HA and COX/PG may also, at least in part, be due to the expression or activation of HAS isoforms (HAS1-3), the expression pattern of prostanoid receptors, or the amount of concomitant stimuli by other inflammatory mediators such as IL-1. For example, isoforms of HAS or EP receptors are expressed and function differentially among tissues and cells. ${ }^{8,39}$ Elucidation of the interaction between HA and COX systems in articular chondrocytes would give important information about the clinical application of HA with simultaneous clinical use of NSAIDs, and may clarify the possible beneficial effects of HA with larger molecular weight in this regard.

\section{Conclusions}

Although articular injection of HA is frequently used in the treatment of arthritic conditions, the precise mechanism by which HA suppresses degradation of cartilage or intraarticular inflammation is still under vigorous investigation. A disease-modifying effect of HA has been suggested by some in vitro studies; however, there has been little clinical evidence thus far. Understanding the wide array of signaling pathways of anti-inflammatory effects by HA will encourage novel anti-degradation strategies for both inflammatory and degenerative arthritides, using HA in combination with other disease-modifying anti-rheumatic drugs and/or NSAIDs.

\section{Disclosure}

None of the authors have any conflict of interest regarding the contents of this article. 


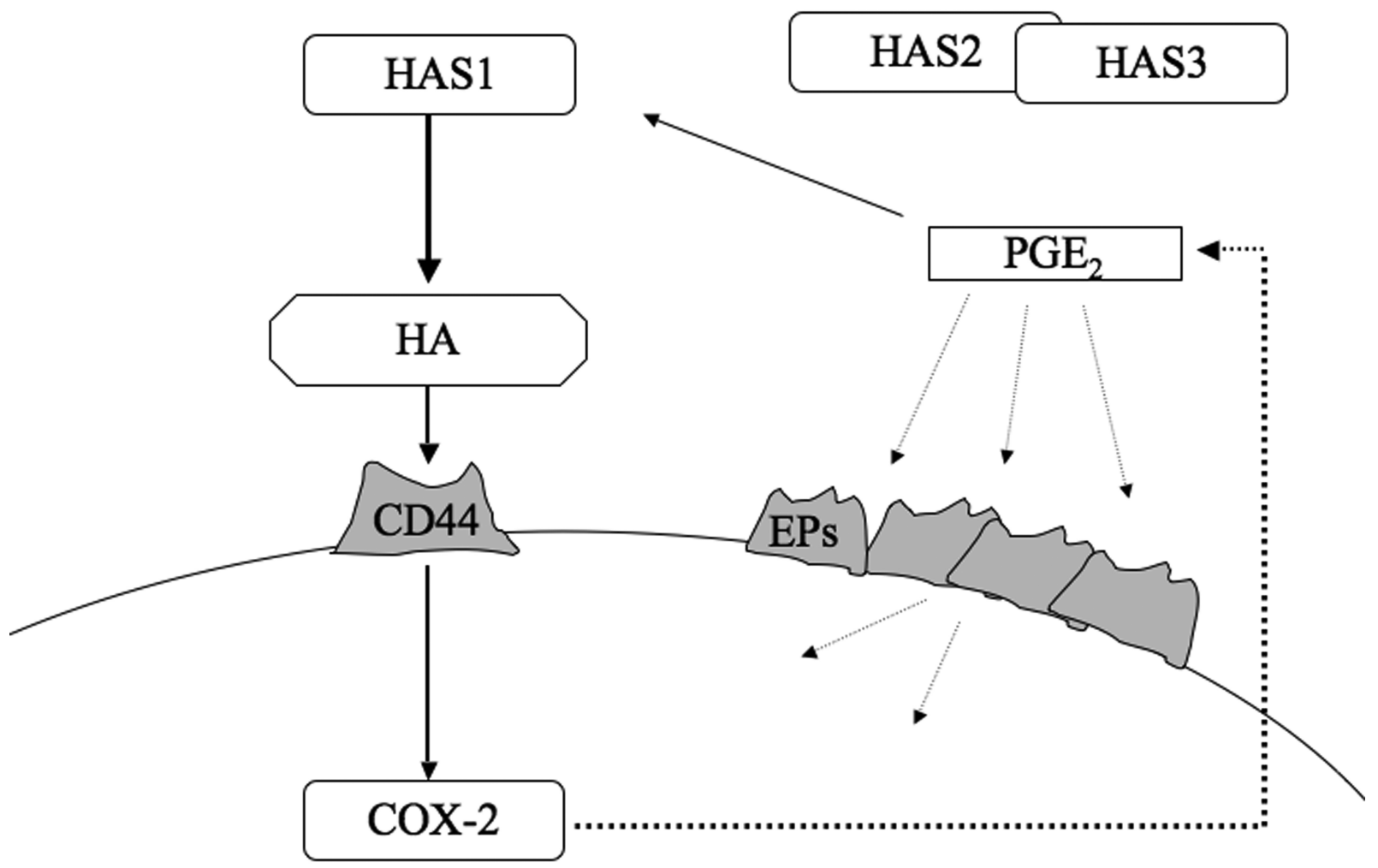

Figure I Possible crosstalk between HA and COX systems. HA may upregulate the inducible expression of COX-2 via CD44.The activated COX-2 induces prostanoids, and $\mathrm{PGE}_{2}$ might further modulate the expressions of HAS and/or COX, through EP receptor(s) at least in part.

Abbreviations: $\mathrm{HAS}$, hyaluronic acid synthase; HA, hyaluronic acid; COX, cyclooxygenase; $P G$, prostaglandin; $E_{\text {, }}$ PGE ${ }_{2}$ receptors.

\section{References}

1. Simon LS, Strand V. The pharmacologic treatment of osteoarthritis. In: Osteoarthritis, Moskowitz RW, Altman RD, Buckwalter JA, Goldberg VM, Hochberg MC, editors. Philadelphia, PA: Lippincott Williams and Willkins; 2007. p. 267-286.

2. Zhang W, Moskowitz RW, Nuki G, et al. OARSI recommendations for the management of hip and knee osteoarthritis, Part II: OARSI evidence-based, expert consensus guidelines. Osteoarthritis Cartilage. 2008;16(2):137-162.

3. Neustadt D, Altman RD. Intra-articular therapy. In: Osteoarthritis, Moskowitz RW, Altman RD, Buckwalter JA, Goldberg VM, Hochberg MC, editors. Philadelphia, PA: Lippincott Williams and Willkins; 2007. p. 287-301.

4. Matsuno H, Yudoh K, Kondo M, Goto M, Kimura T. Biochemical effect of intra-articular injections of high molecular weight hyaluronate in rheumatoid arthritis patients. Inflamm Res. 1999;48(3):154-159.

5. Goto M, Hanyu T, Yoshio T, et al. Intra-articular injection of hyaluronate (SI-6601D) improves joint pain and synovial fluid prostaglandin E2 levels in rheumatoid arthritis: a multicenter clinical trial. Clin Exp Rheumatol. 2001;19(4):377-383.

6. Roth A, Mollenhauer J, Wagner A, et al. Intra-articular injections of high-molecular-weight hyaluronic acid have biphasic effects on joint inflammation and destruction in rat antigen-induced arthritis. Arthritis Res Ther. 2005;7(3):R677-R686.

7. Noble PW, McKee CM, Cowman M, Shin HS. Hyaluronan fragments activate an NF-kappa B/I-kappa B alpha autoregulatory loop in murine macrophages. J Exp Med. 1996;183(5):2373-2378.

8. Itano N, Sawai T, Yoshida M, et al. Three isoforms of mammalian hyaluronan synthases have distinct enzymatic properties. $J$ Biol Chem. 1999;274(35):25085-25092.
9. Aihara S, Murakami N, Ishii R, et al. [Effects of sodium hyaluronate on the nociceptive response of rats with experimentally induced arthritis]. Nippon Yakurigaku Zasshi. 1992;100(4):359-365.

10. Gotoh S, Onaya J, Abe M, et al. Effects of the molecular weight of hyaluronic acid and its action mechanisms on experimental joint pain in rats. Ann Rheum Dis. 1993;52(11):817-822.

11. Taylor KR, Gallo RL. Glycosaminoglycans and their proteoglycans: host-associated molecular patterns for initiation and modulation of inflammation. FASEB J. 2006;20(1):9-22.

12. Wang KX, Denhardt DT. Osteopontin: role in immune regulation and stress responses. Cytokine Growth Factor Rev. 2008;19(5-6): 333-345.

13. Naor D, Sionov RV, Ish-Shalom D. CD44: structure, function, and association with the malignant process. Adv Cancer Res. 1997;71: 241-319.

14. Lesley J, Hyman R, Kincade PW. CD44 and its interaction with extracellular matrix. Adv Immunol. 1993;54:271-335.

15. Aruffo A, Stamenkovic I, Melnick M, Underhill CB, Seed B. CD44 is the principal cell surface receptor for hyaluronate. Cell. 1990;61(7):1303-1313.

16. Weber GF, Ashkar S, Glimcher MJ, Cantor H. Receptor-ligand interaction between CD44 and osteopontin (Eta-1). Science. 1996;271(5248):509-512.

17. Shimazu A, Jikko A, Iwamoto M, et al. Effects of hyaluronic acid on the release of proteoglycan from the cell matrix in rabbit chondrocyte cultures in the presence and absence of cytokines. Arthritis Rheum. 1993;36(2):247-253.

18. Lisignoli G, Grassi F, Zini N, et al. Anti-Fas-induced apoptosis in chondrocytes reduced by hyaluronan: evidence for CD44 and CD54 (intercellular adhesion molecule 1) invovement. Arthritis Rheum. 2001;44(8):1800-1807. 
19. Aigner T, Kurz B, Fukui N, Sandell L. Roles of chondrocytes in the pathogenesis of osteoarthritis. Curr Opin Rheumatol. 2002;14(5): 578-584.

20. Pelletier J, Martel-Pelletier J, Abramson S. Osteoarthritis, an inflammatory disease. Arthritis Rheum. 2001;44(6):1237-1247.

21. Chockalingam PS, Zeng W, Morris EA, Flannery CR. Release of hyaluronan and hyaladherins (aggrecan G1 domain and link proteins) from articular cartilage exposed to ADAMTS-4 (aggrecanase 1) or ADAMTS-5 (aggrecanase 2). Arthritis Rheum. 2004;50(9): 2839-2848.

22. Kawasaki K, Ochi M, Uchio Y, Adachi N, Matsusaki M. Hyaluronic acid enhances proliferation and chondroitin sulfate synthesis in cultured chondrocytes embedded in collagen gels. J Cell Physiol. 1999;179(2):142-148.

23. Frean SP, Abraham LA, Lees P. In vitro stimulation of equine articular cartilage proteoglycan synthesis by hyaluronan and carprofen. Res Vet Sci. 1999;67(2):183-190.

24. Takahashi K, Goomer RS, Harwood F, Kubo T, Hirasawa Y, Amiel D. The effects of hyaluronan on matrix metalloproteinase-3 (MMP-3), interleukin-1beta(IL-1beta), and tissue inhibitor of metalloproteinase-1 (TIMP-1) gene expression during the development of osteoarthritis. Osteoarthritis Cartilage. 1999;7(2):182-190.

25. Yatabe T, Mochizuki S, Takizawa M, et al. Hyaluronan inhibits expression of ADAMTS4 (aggrecanase-1) in human osteoarthritic chondrocytes. Ann Rheum Dis. 2009 Jan 5. [Epub ahead of print]

26. Karna E, Miltyk W, Palka JA, Jarzabek K, Wolczynski S. Hyaluronic acid counteracts interleukin-1-induced inhibition of collagen biosynthesis in cultured human chondrocytes. Pharmacol Res. 2006;54(4):275-281.

27. Julovi S, Yasuda T, Shimizu M, Hiramatsu T, Nakamura T. Inhibition of interleukin-1beta-stimulated production of matrix metalloproteinases by hyaluronan via CD44 in human articular cartilage. Arthritis Rheum. 2004;50(2):516-525.

28. Tanaka M, Masuko-Hongo K, Kato T, Nishioka K, Nakamura H. Suppressive effects of hyaluronan on MMP-1 and RANTES production from chondrocytes. Rheumatol Int. 2006;26(3):185-190.

29. Hiramitsu T, Yasuda $\mathrm{T}$, Ito $\mathrm{H}$, et al. Intercellular adhesion molecule-1 mediates the inhibitory effects of hyaluronan on interleukin-1betainduced matrix metalloproteinase production in rheumatoid synovial fibroblasts via down-regulation of NF-kappaB and p38. Rheumatology (Oxford). 2006;45(7):824-832.
30. Nonaka T, Kikuchi H, Ikeda T, Okamoto Y, Hamanishi C, Tanaka S. Hyaluronic acid inhibits the expression of u-PA, PAI-1, and u-PAR in human synovial fibroblasts of osteoarthritis and rheumatoid arthritis. J Rheumatol. 2000;27(4):997-1004.

31. Hsieh YS, Yang SF, Lue KH, Chu SC, Lu KH. Effects of different molecular weight hyaluronan products on the expression of urokinase plasminogen activator and inhibitor and gelatinases during the early stage of osteoarthritis. J Orthop Res. 2008;26(4):475-484.

32. Dunlop ME, Muggli EE. Hyaluronan increases glomerular cyclooxygenase-2 protein expression in a p38 MAP-kinase-dependent process Kidney Int. 2002;61(5):1729-1738.

33. Mitsui Y, Gotoh M, Nakama K, Yamada T, Higuchi F, Nagata K. Hyaluronic acid inhibits mRNA expression of proinflammatory cytokines and cyclooxygenase-2/prostaglandin E(2) production via CD44 in interleukin-1-stimulated subacromial synovial fibroblasts from patients with rotator cuff disease. J Orthop Res. 2008;26(7):1032-1037.

34. Misra S, Obeid L M, Hannun YA, et al. Hyaluronan constitutively regulates activation of COX-2-mediated cell survival activity in intestinal epithelial and colon carcinoma cells. J Biol Chem. 2008;283(21): 14335-14344.

35. Sun LK, Wahl P, Bilic G, Wuthrich RP. CD44-mediated cyclooxygenase-2 expression and thromboxane A2 production in RAW 264.7 macrophages. Inflamm Res. 2001;50(10):496-499.

36. Murphy JF, Lennon F, Steele C, Kelleher D, Fitzgerald D, Long AC. Engagement of CD44 modulates cyclooxygenase induction, VEGF generation, and proliferation in human vascular endothelial cells. FASEB J. 2005;19(3):446-448.

37. Stuhlmeier KM. Prostaglandin E2: a potent activator of hyaluronan synthase 1 in type-B-synoviocytes. Biochim Biophys Acta. 2007;1770(1):121-129.

38. Kinugasa Y, Hatori M, Ito H, Kurihara Y, Ito D, Nagumo M. Inhibition of cyclooxygenase-2 suppresses invasiveness of oral squamous cell carcinoma cell lines via down-regulation of matrix metalloproteinase-2 and CD44. Clin Exp Metastasis. 2004;21(8):737-745.

39. Sugimoto Y, Narumiya S. Prostaglandin E receptors. J Biol Chem. 2007;282(16):11613-11617.
International Journal of General Medicine

\section{Publish your work in this journal}

The International Journal of General Medicine is an international, peer-reviewed open-access journal that focuses on general and internal medicine, pathogenesis, epidemiology, diagnosis, monitoring and treatment protocols. The journal is characterized by the rapid reporting of reviews, original research and clinical studies across all disease areas.

\section{Dovepress}

A key focus is the elucidation of disease processes and management protocols resulting in improved outcomes for the patient.The manuscript management system is completely online and includes a very quick and fair peer-review system. Visit http://www.dovepress.com/ testimonials.php to read real quotes from published authors. 